\title{
Article
}

Mycosphere

Doi 10.5943/mycosphere/8/2/11

Copyright $\odot$ Guizhou Academy of Agricultural Sciences

\section{Two new endophytic species of Phyllosticta (Phyllostictaceae, Botryosphaeriales) from Southern China}

\author{
Lin $S^{1,2}$, Sun $X^{3}$, He $W^{1}$, Zhang $Y^{2}$ \\ ${ }^{1}$ Beijing Key Laboratory for Forest Pest Control, Beijing Forestry University, Beijing 100083, PR China \\ ${ }^{2}$ Institute of Microbiology, P.O. Box 61, Beijing Forestry University, Beijing 100083, PR China \\ ${ }^{3}$ State Key Laboratory of Mycology, Institute of Microbiology, Chinese Academy of Sciences, Beijing 100101, China
}

Lin S, Sun X, He W, Zhang Y 2017 - Two new endophytic species of Phyllosticta (Phyllostictaceae, Botryosphaeriales) from Southern China. Mycosphere 8(2), 1273-1288, Doi 10.5943/mycosphere/8/2/11

\begin{abstract}
Phyllosticta is an important genus known to cause various leaf spots and fruit diseases worldwide on a large range of hosts. Two new endophytic species of Phyllosticta (P. dendrobii and $P$. illicii) are described and illustrated from Dendrobium nobile and Illicium verum in China. Phylogenetic analysis based on combined ITS, LSU, tefl-a, ACT and GPDH loci supported their separation from other species of Phyllosticta. Morphologically, P. dendrobii is most comparable with $P$. aplectri, while the large-sized pycnidia of $P$. dendrobii differentiate it from $P$. aplectri. Members of Phyllosticta are first reported from Dendrobium and Illicium.
\end{abstract}

Key words - Asia - Botryosphaeriales - leaf spots - Multilocus phylogeny

\section{Introduction}

Phyllosticta Pers. was introduced by Persoon (1818) and typified by P. convallariae Pers. Many species of Phyllosticta cause leaf and fruit spots on various host plants, such as $P$. citricarpa (McAlpine) Aa, which causes citrus black spot (Baayen et al. 2002, Glienke et al. 2011), P. ampelicida (Engelm.) Aa species complex that causes black rot disease on grapevines (Wicht et al. 2012), and the P. musarum (Cooke) Aa species complex that causes banana freckle disease (Pu et al. 2008, Wong et al. 2012). Some species of Phyllosticta have also been isolated as endophytes from a wide range of hosts, and other species are regarded as saprobes, e.g. P. capitalensis Henn., P. carpogena (Shear) Aa and P. ericae Allesch. (van der Aa 1973, Baayen et al. 2002, van der Aa \& Vanev 2002, Glienke et al. 2011, Wikee et al. 2011, 2013).

The generic concept of Phyllosticta has undergone substantial changes since its establishment in 1818. Allescher (1898) considered Phyllosticta as Phoma-like, but as foliar pathogens, and Phoma Sacc. on other plant parts, and Grove (1935) regarded Phyllosticta as a parasite and Phoma as saprobe or wound parasite. Host association has been used in separating "Phyllosticta" species (Seaver 1922, Grove 1935), which has been widely followed in the $20^{\text {th }}$ century, and led to the introduction of numerous species names. Presently there are approximately 3,213 epithets known for Phyllosticta (http://www.indexfungorum.org, accessed August 2017), but many of these reflect old concepts of the genus, and have been accommodated elsewhere (van der Aa \& Vanev 2002). Phyllosticta s. str. was first monographed by van der Aa (1973), who described and illustrated 46 species, and listed the sexual morphs for 12 species, and the spermatial morphs for 17 based mostly on material collected in Europe and North America. More recently, van der Aa \& Vanev (2002) 
revised all species described in Phyllosticta, and accepted 190 epithets. A multi-locus phylogeny of 129 isolates of Phyllosticta, representing about 170 species names indicated that Phyllosticta is sister to the Botryosphaeriaceae (Botryosphaeriales, Dothideomycetes) and that many species are synonyms of the cosmopolitan P. capitalensis (Wikee et al. 2013). The family Phyllostictaceae was first proposed by Fries (1849) and resurrected by Wikee et al. (2013) to accommodate Phyllosticta. The sexual stage of Phyllosticta is Guignardia Viala \& Ravaz, and the earlier and widely used name Phyllosticta was chosen over Guignardia (Glienke et al. 2011, Sultan et al. 2011, Wikee et al. 2011, 2013, Wong et al. 2012).

Dendrobium nobile Lindley is a member of the family Orchidaceae, which is a fundamental herb used in traditional Chinese medicine. Illicium verum Hook. $\mathrm{f}$. is an aromatic evergreen tree of the family Illiciaceae, the fruit of which (Chinese star anise) has long been used in traditional Chinese medicine and the food industry with the actions of dispelling cold, regulating the flow of Qi and relieving pain. Both Dendrobium nobile and Illicium verum are native to China. During a survey of endophytic fungi associated with D. nobile and I. verum in China, two Phyllosticta species were isolated. Based on morphological and molecular phylogenetic inferences, we introduce them as $P$. dendrobii and $P$. illicii respectively.

\section{Material \& Methods}

\section{Fungal isolation}

Wood segments of $5 \mathrm{~mm} \times 5 \mathrm{~mm} \times 2 \mathrm{~mm}$ were cut from the healthy tissue, and washed in tap water and wiped with $70 \%$ ethanol. They were suspended in $70 \%$ ethanol for $15 \mathrm{~min}$, and washed in distilled water (three times) before placing on MEA. All plates were incubated at $28^{\circ} \mathrm{C}$. The growing tips of hyphae of Phyllosticta colonies that developed were cut out and transferred to fresh MEA plates.

\section{Morphological study of fungi}

Growth rates and culture characteristics of the isolates were determined at $28{ }^{\circ} \mathrm{C}$ on MEA. Fungal structures (conidia, conidiogenous cells etc.) were mounted in water for observation with a microscope (Nikon Eclipse E600). Nomenclatural novelties and descriptions were deposited in MycoBank (www.mycobank.org, Crous et al. 2004) and Faces Of Fungi (http://www.facesoffungi.org/, Jayasiri et al. 2015). Type specimens were deposited in Mycological Herbarium of the Institute of Microbiology, Chinese Academy of Sciences, Beijing, China (HMAS), with extype living cultures deposited in China General Microbiological Culture Collection Center (CGMCC). At least 30 pycnidia, conidiogenous cells or conidia were measured to calculate the mean dimensions and standard deviations (SD) given in the formal descriptions.

\section{DNA extraction, PCR amplification, and sequencing}

Isolates for DNA extraction were grown on MEA, and total genomic DNA extracted from mycelia with CTAB plant genome DNA fast extraction kit (Aidlab Biotechnologies Co., Ltd, Beijing, China). Internal transcribed spacer (ITS), Actin (ACT), translation elongation factor 1alpha (tefl- $\alpha$ ), 28S large subunit ribosomal RNA gene (LSU), and glyceraldehyde-3-phosphate dehydrogenase (GPDH) genes were amplified with primer pairs ITS1/ITS4 (White et al. 1990), ACT512F/ACT783R (Carbone \& Kohn 1999), EF1-728F/EF1-786R (Carbone \& Kohn 1999), LROR/LR5 (Moncalvo et al. 1995) and GDF1/Gpd2-LM (Myllys et al. 2002, Guerber et al. 2003) respectively.

\section{Sequence alignment and phylogenetic analysis}

Sequence data of combined ITS, ACT, tefl- $\alpha$, LSU and GPDH loci were used to infer the phylogenetic relationships of $P$. dendrobium, $P$. illicium and other reported Phyllosticta species for which confirmed sequence data were available from GenBank (http://www.ncbi.nlm.nih.gov/genbank/). A multiple alignment was done in MEGA v. 6 (Tamura et 
al. 2013) and analyses were performed in PAUP v. 4.0b10 (Swofford 2002) and MrBayes v. 3.1.2. (Ronquist \& Huelsenbeck 2003). Prior to phylogenetic analysis, ambiguous sequences at the start and the end were deleted and gaps manually adjusted to optimize alignment. Maximum Likelihood bootstrap values (ML-BS) obtained from 1000 replicates were performed using raxml GUI 1.31 (Michalak 2012) with the GTRGAMMA and GTRCAT models to assess the reliability of the nodes. Maximum Parsimony (MP) analysis was conducted using heuristic searches as implemented in PAUP, with the default options method. Analyses were done under different parameters of maximum parsimony criteria as outlined in Zhang et al. (2008). Clade stability was assessed in a bootstrap analysis with 1000 replicates, random sequence additions with maxtrees set to 1000 and other default parameters as implemented in PAUP. For the Bayesian analysis, the best-fit model of nucleotide evolution $(\mathrm{GTR}+\mathrm{I}+\mathrm{G})$ was selected by Akaike information criterion (AIC; Posada \& Buckley 2004) in MrModeltest 2.3. The metropolis-coupled Markov Chain Monte Carlo (MCMCMC) approach was used to calculate posterior probabilities (Huelsenbeck \& Ronquist 2005). A preliminary Bayesian inference (BI) analysis using MrBayes revealed that the Markov Chain Monte Carlo steady state was reached after less than 10,000 generations (the average standard deviation of split frequencies was constantly below 0.01). A conservative burn-in of 100 dendrograms was chosen and a full analysis of 5,000,000 generations was carried out with sampling every 100 generations. Trees were viewed in TREEVIEW (Page 1996). The nucleotide sequences reported in this paper were deposited in GenBank, and alignments in TreeBASE (S21576).

\section{Results}

The combined ITS, ACT, tefl-a, LSU and GPDH dataset comprised 114 strains. The dataset consisted of 2521 characters after alignment, and all sites were included in the MP analysis. Of the included bases, 768 sites $(30.5 \%)$ were parsimony-informative. A heuristic search with random addition of taxa (1000 replicates) and treating gaps as missing characters generated 720 equally parsimonious trees. All trees were similar in topology and not significantly different (figures not shown). One of the most parsimonious trees $(\mathrm{TL}=3898, \mathrm{CI}=0.408, \mathrm{RI}=0.780, \mathrm{RC}=0.319)$ is shown in Fig. 1.

\section{Taxonomy}

Phyllosticta dendrobii S. Lin, Y. Zhang ter, sp. nov.

Fig. 2

MycoBank: MB821784; Facesoffungi number: FoF03422

Etymology - Named after the host genus from which it was collected, Dendrobium nobile.

Conidiomata pycnidial, mostly aggregated in clusters, black, erumpent, globose to clavate or elongated with necks up to $700 \mu \mathrm{m}$ long, exuding colourless to opaque conidial masses. Pycnidia up to $280 \mu \mathrm{m}$ diam. Pycnidial wall of several layers of textura angularis, up to $40 \mu \mathrm{m}$ thick, inner wall of hyaline textura angularis. Ostiole central, up to $20 \mu \mathrm{m}$ diam. Conidiophores reduced to conidiogenous cells, Conidiogenous cells terminal, subcylindrical, hyaline, smooth, 6-10 × 3-4 $\mu \mathrm{m}$, proliferating several times percurrently near apex. Conidia $(5-) 8-9(-10) \times 5(-7) \mu \mathrm{m}$, solitary, hyaline, aseptate, thin and smooth walled, coarsely guttulate, or with a single large central guttule, ovoid to irregularly ellipsoid, enclosed in a thin mucoid sheath, 1-2 $\mu \mathrm{m}$ thick, and bearing a hyaline, apical mucoid appendage, $(4-) 6-9(-10) \times 2(-3) \mu \mathrm{m}$, flexible, unbranched, tapering towards an acutely rounded tip. After 2 months, mucoid sheath and apical mucoid appendage disappear. Ascomata similar to conidiomata in general anatomy. Asci bitunicate, hyaline, clavate to broadly fusoid-ellipsoid, 54-95 $\times 8-13 \mu \mathrm{m}$, with visible apical chamber, $2 \mu \mathrm{m}$ diam. Ascospores bito multi-seriate, hyaline, smooth, granular to guttulate, aseptate, straight and slightly curved, widest in the middle, limoniform with obtuse ends, $(12-) 14-16(-18) \times 3-6 \mu \mathrm{m}$, with distinct hyaline gelatinous caps at both ends, mostly less than $10 \mu \mathrm{m}$, seldom over $15 \mu \mathrm{m}$ long. 
Table 1. Phyllosticta isolates investigated in this study.

\begin{tabular}{|c|c|c|c|c|c|c|c|c|}
\hline \multirow{2}{*}{ Species } & \multirow{2}{*}{ Culture } & \multirow{2}{*}{ Host } & \multirow{2}{*}{ Country } & \multicolumn{5}{|c|}{ GenBank Number } \\
\hline & & & & ITS & LSU & tef1-a & ACT & GPDH \\
\hline $\begin{array}{l}\text { Botryosphaeria } \\
\text { obtusa }\end{array}$ & CMW 8232 & Conifers & South Africa & AY972105 & N/A & DQ280419 & AY972111 & N/A \\
\hline $\begin{array}{l}\text { Guignardia } \\
\text { gaultheriae }\end{array}$ & CBS 447.70 & $\begin{array}{l}\text { Gaultheria } \\
\text { humifusa }\end{array}$ & USA & JN692543 & KF206298 & JN692531 & KF289248 & JN692508 \\
\hline \multirow[t]{2}{*}{ G. mangiferae } & IMI 260.576 & Mangifera indica & India & JF261459 & KF206222 & JF261501 & JF343641 & JF343748 \\
\hline & CPC 20260 & Arecaceae & Thailand & KF206193 & KF206243 & KF289187 & KF289294 & KF289114 \\
\hline G. musicola & CBS123405 & Musa acuminata & Thailand & FJ538334 & N/A & FJ538392 & FJ538450 & N/A \\
\hline G. rhodorae & CBS 901.69 & Rhododendron & Netherlands & KF206174 & KF206292 & KF289230 & KF289256 & KF289166 \\
\hline \multicolumn{9}{|l|}{ abieticola } \\
\hline P. alliacea & MUCC0014 & Allium fistulosum & Japan & AB454263 & N/A & N/A & N/A & N/A \\
\hline \multirow{2}{*}{ P. aloeicola } & CPC 21020 & Aloe ferox & South Africa & KF154280 & KF206214 & KF289193 & KF289311 & KF289124 \\
\hline & CPC 21021 & Aloe ferox & South Africa & KF154281 & KF206213 & KF289194 & KF289312 & KF289125 \\
\hline P. ampelicida & ATCC200578 & Vitis riparia & USA & KC193586 & N/A & N/A & KC193581 & KC193584 \\
\hline P. ardisiicola & NBRC102261 & Ardisia crenata & Japan & AB454274 & N/A & N/A & $\mathrm{N} / \mathrm{A}$ & N/A \\
\hline P. aspidistricola & NBRC102244 & Aspidistra elatior & Japan & AB454260 & N/A & N/A & N/A & N/A \\
\hline P. beaumarisii & CBS 535.87 & $\begin{array}{l}\text { Muehlenbekia } \\
\text { adpressa }\end{array}$ & Australia & AY042927 & KF306229 & KF289170 & KF306232 & KF289074 \\
\hline \multirow[t]{2}{*}{ P. bifrenariae } & CBS 128855 & $\begin{array}{l}\text { Bifrenaria } \\
\text { harrisoniae }\end{array}$ & Brazil & JF343565 & KF206209 & JF343586 & JF343649 & JF343744 \\
\hline & CPC 17467 & $\begin{array}{l}\text { Bifrenaria } \\
\text { harrisoniae }\end{array}$ & Brazil & KF170299 & KF206260 & KF289207 & KF289283 & KF289138 \\
\hline \multirow[t]{2}{*}{ P. brazillianiae } & CBS 126270 & Mangifera indica & Brazil & JF343572 & KF206217 & JF343593 & JF343656 & JF343758 \\
\hline & LGMF 333 & Mangifera indica & Brazil & JF343574 & KF206216 & JF343595 & JF343658 & JF343760 \\
\hline \multirow[t]{2}{*}{ P. capitalensis } & CPC 18848 & $\begin{array}{l}\text { Stanhopea } \\
\text { graveolens }\end{array}$ & Brazil & JF261465 & KF206255 & JF261507 & KF289289 & JF343776 \\
\hline & CPC 20267 & $\begin{array}{l}\text { Baccaurea } \\
\text { ramiflora }\end{array}$ & Brazil & KF206195 & KF206237 & KF289173 & KF306233 & KF289078 \\
\hline P. carochlae & $\begin{array}{l}\text { CGMCC } \\
3.17317\end{array}$ & $\begin{array}{l}\text { Caryota } \\
\text { ochlandra }\end{array}$ & China & KJ847422 & N/A & KJ847444 & KJ847430 & KJ847438 \\
\hline
\end{tabular}




\begin{tabular}{|c|c|c|c|c|c|c|c|c|}
\hline & $\begin{array}{l}\text { CGMCC } \\
3.17318\end{array}$ & $\begin{array}{l}\text { Caryota } \\
\text { ochlandra }\end{array}$ & China & KJ847423 & N/A & KJ847445 & KJ847431 & KJ847439 \\
\hline \multirow[t]{2}{*}{ P. cavendishii } & BRIP554196 & $\begin{array}{l}\text { Musa } \\
\text { Formosana }\end{array}$ & Taiwan & JQ743562 & N/A & KF009743 & KF014080 & N/A \\
\hline & BRIP58008 & Banana & Australia & KC988365 & N/A & KF009742 & KF014071 & N/A \\
\hline \multirow[t]{2}{*}{ P. citriasiana } & CBS 120486 & Citrus maxima & Thailand & FJ538360 & KF206314 & FJ538418 & FJ538476 & JF343686 \\
\hline & CBS 123371 & Citrus maxima & Vietnam & FJ538356 & KF206309 & FJ538414 & FJ538472 & JF343690 \\
\hline \multirow[t]{2}{*}{ P. citribraziliensis } & CBS 100098 & Citrus limon & Brazil & FJ538352 & KF206221 & FJ538410 & FJ538468 & JF343691 \\
\hline & СРC 17464 & Citrus sp. & Brazil & KF170300 & KF206263 & KF289224 & KF289280 & KF289159 \\
\hline \multirow[t]{2}{*}{ P. citricarpa } & CBS 127454 & Citrus limon & Australia & JF343583 & KF206306 & JF343604 & JF343667 & JF343771 \\
\hline & CBS 127455 & Citrus sinensis & Australia & JF343584 & KF206305 & JF343605 & JF343668 & JF343772 \\
\hline \multirow[t]{2}{*}{ P. citrichinaensis } & $\begin{array}{l}\text { ZJUCC } \\
200956\end{array}$ & Citrus reticulata & China & JN791620 & N/A & JN791459 & JN791533 & N/A \\
\hline & ZJUCC 200964 & Citrus maxima & China & JN791611 & N/A & JN791461 & JN791535 & N/A \\
\hline P. citrimaxima & CPC 20276 & Citrus maxima & Thailand & KF170304 & KF206229 & KF289222 & KF289300 & KF289157 \\
\hline \multirow[t]{2}{*}{ P. concentrica } & CBS 937.70 & Hedera helix & Italy & FJ538350 & KF206291 & FJ538408 & KF289257 & JF411745 \\
\hline & CBS 134749 & Hedera sp. & Spain & KF170310 & KF206256 & KF289228 & KF289288 & KF289163 \\
\hline \multirow[t]{2}{*}{ P. cordylinophila } & CPC 20261 & $\begin{array}{l}\text { Cordyline } \\
\text { fruticosa }\end{array}$ & Thailand & KF170287 & KF206242 & KF289172 & KF289295 & KF289076 \\
\hline & CРC 20277 & $\begin{array}{l}\text { Cordyline } \\
\text { fruticosa }\end{array}$ & Thailand & KF170288 & KF206228 & KF289171 & KF289301 & KF289075 \\
\hline P. cornicola & CBS 111639 & Cornus florida & USA & KF170307 & N/A & N/A & KF289234 & N/A \\
\hline \multirow[t]{2}{*}{ P. cussonia } & CPC 14873 & Cussonia sp. & South Africa & JF343578 & KF206279 & JF343599 & JF343662 & JF343764 \\
\hline & СРC 14875 & Cussonia sp. & South Africa & JF343579 & KF206278 & JF343600 & JF343663 & JF343765 \\
\hline \multirow[t]{3}{*}{ P. dendrobii } & $\begin{array}{l}\text { CGMCC } \\
3.18665\end{array}$ & $\begin{array}{l}\text { Dendrobium } \\
\text { nobile }\end{array}$ & China & MF180192 & MF180209 & N/A & MF180218 & MF180227 \\
\hline & $\begin{array}{l}\text { CGMCC } \\
3.18666\end{array}$ & $\begin{array}{l}\text { Dendrobium } \\
\text { nobile }\end{array}$ & China & MF180193 & MF180210 & MF180201 & MF180219 & MF180228 \\
\hline & $\begin{array}{l}\text { CGMCC } \\
3.18667\end{array}$ & $\begin{array}{l}\text { Dendrobium } \\
\text { nobile }\end{array}$ & China & MF180194 & MF180211 & MF180202 & MF180220 & MF180229 \\
\hline P. elongata & CBS 126.22 & $\begin{array}{l}\text { Oxycoccus } \\
\text { macrocarpos }\end{array}$ & USA & FJ538353 & N/A & FJ538411 & FJ538469 & KF289164 \\
\hline P. ericarum & CBS 132534 & Erica gracilis & South Africa & KF206170 & KF206253 & KF289227 & KF289291 & KF289162 \\
\hline P. eugeniae & CBS 445.82 & Eugenia & Indonesia & AY042926 & KF206288 & KF289208 & KF289246 & KF289139 \\
\hline
\end{tabular}




\begin{tabular}{|c|c|c|c|c|c|c|c|c|}
\hline \multirow[b]{2}{*}{ P. fallopiae } & \multirow[b]{2}{*}{ MUCC0113 } & \multicolumn{2}{|l|}{ aromatica } & \multirow[b]{2}{*}{ AB454307 } & \multirow[b]{2}{*}{ N/A } & \multirow[b]{2}{*}{ N/A } & \multirow[b]{2}{*}{ N/A } & \multirow[b]{2}{*}{ N/A } \\
\hline & & $\begin{array}{l}\text { Fallopia } \\
\text { japonica }\end{array}$ & Japan & & & & & \\
\hline P. gaultheriae & CBS 447.70 & $\begin{array}{l}\text { Gaultheria } \\
\text { humifusa }\end{array}$ & USA & JN692543 & KF206298 & JN692531 & KF289248 & JN692508 \\
\hline \multirow[t]{2}{*}{ P. foliorum } & CBS 174.77 & $\begin{array}{l}\text { Cryptomeria } \\
\text { japonica }\end{array}$ & USA & KF170308 & KF206290 & KF289200 & KF289245 & KF289131 \\
\hline & CBS 447.68 & Taxus baccata & Netherlands & KF170309 & KF206287 & KF289201 & KF289247 & KF289132 \\
\hline P. hamamelidis & MUCC 149 & $\begin{array}{l}\text { Hamamelis } \\
\text { japonica }\end{array}$ & Japan & KF170289 & N/A & N/A & KF289309 & N/A \\
\hline \multirow[t]{2}{*}{ P. hostae } & $\begin{array}{l}\text { CGMCC } \\
3.14355\end{array}$ & $\begin{array}{l}\text { Hosta } \\
\text { plantaginea }\end{array}$ & China & JN692535 & N/A & JN692524 & JN692512 & JN692504 \\
\hline & $\begin{array}{l}\text { CGMCC } \\
3.14356\end{array}$ & $\begin{array}{l}\text { Hosta } \\
\text { plantaginea }\end{array}$ & China & JN692536 & N/A & JN692525 & JN692513 & JN692505 \\
\hline \multirow[t]{2}{*}{ P. hubeiensis } & $\begin{array}{l}\text { CGMCC } \\
3.14986\end{array}$ & $\begin{array}{l}\text { Viburnum } \\
\text { odoratissimim }\end{array}$ & China & JX025037 & N/A & JX025042 & JX025032 & JX025027 \\
\hline & $\begin{array}{l}\text { CGMCC } \\
3.14987\end{array}$ & $\begin{array}{l}\text { Viburnum } \\
\text { odoratissimim }\end{array}$ & China & JX025038 & N/A & JX025043 & JX025033 & JX025028 \\
\hline \multirow[t]{2}{*}{ P. hymenocallidicola } & CBS 131309 & $\begin{array}{l}\text { Hymenocallis } \\
\text { littoralis }\end{array}$ & Australia & JQ044423 & JQ044443 & KF289211 & KF289242 & KF289142 \\
\hline & СРC 19331 & $\begin{array}{l}\text { Hymenocallis } \\
\text { littoralis }\end{array}$ & Australia & KF170303 & KF206254 & KF289212 & KF289290 & KF289143 \\
\hline \multirow[t]{2}{*}{ P. hypoglossi } & CBS 101.72 & Ruscus aculeatus & Italy & FJ538365 & KF206326 & FJ538423 & FJ538481 & JF343694 \\
\hline & CBS 434.92 & Ruscus aculeatus & Italy & FJ538367 & KF206299 & FJ538425 & FJ538483 & JF343695 \\
\hline \multirow[t]{2}{*}{ P. ilicis-aquifolii } & $\begin{array}{l}\text { CGMCC } \\
\mathbf{3 . 1 4 3 5 8}\end{array}$ & Ilex aquifolium & China & JN692538 & N/A & JN692526 & JN692514 & N/A \\
\hline & $\begin{array}{l}\text { CGMCC } \\
3.14359\end{array}$ & Ilex aquifolium & China & JN692539 & N/A & JN692527 & JN692515 & N/A \\
\hline \multirow[t]{3}{*}{ P. illicii } & $\begin{array}{l}\text { CGMCC } \\
3.18670\end{array}$ & Illicium verum & China & MF180195 & MF180212 & MF180203 & MF180221 & N/A \\
\hline & $\begin{array}{l}\text { CGMCC } \\
3.18672\end{array}$ & Illicium verum & China & MF180196 & MF180213 & MF180204 & MF180222 & N/A \\
\hline & $\begin{array}{l}\text { CGMCC } \\
3.18673\end{array}$ & Illicium verum & China & MF180197 & MF180214 & MF180205 & MF180223 & N/A \\
\hline
\end{tabular}




\begin{tabular}{|c|c|c|c|c|c|c|c|c|}
\hline & CGMCC & Illicium verum & China & MF180198 & MF180215 & MF180206 & MF180224 & N/A \\
\hline & 3.18674 & & & & & & & \\
\hline & CGMCC & Illicium verum & China & MF180199 & MF180216 & MF180207 & MF180225 & N/A \\
\hline & 3.18675 & & & & & & & \\
\hline & CGMCC & Illicium verum & China & MF180200 & MF180217 & MF180208 & MF180226 & N/A \\
\hline & 3.18676 & & & & & & & \\
\hline & CGMCC & Illicium verum & China & MF198233 & MF198238 & N/A & MF198241 & N/A \\
\hline & 3.18668 & & & & & & & \\
\hline & CGMCC & Illicium verum & China & MF198234 & MF198239 & MF198236 & MF198242 & N/A \\
\hline & 3.18669 & & & & & & & \\
\hline & CGMCC & Illicium verum & China & MF198235 & MF198240 & MF198237 & MF198243 & N/A \\
\hline & 3.18671 & & & & & & & \\
\hline P. kerriae & MAFF240047 & Kerria japonica & Japan & AB454266 & N/A & N/A & N/A & N/A \\
\hline P. leucothoicola & CBS 136073 & $\begin{array}{l}\text { Leucothoe } \\
\text { catesbaei }\end{array}$ & Japan & AB454370 & AB454370 & N/A & KF289310 & N/A \\
\hline P. ligustricola & MUCC0024 & $\begin{array}{l}\text { Ligustrum } \\
\text { obtusifolium }\end{array}$ & Japan & AB454269 & N/A & N/A & AB704212 & N/A \\
\hline P. maculata & CPC18347 & $\begin{array}{l}\text { Musa cv.Goly- } \\
\text { goly pot-pot }\end{array}$ & Australia & JQ743570 & N/A & KF009700 & KF014016 & N/A \\
\hline & BRIP46622 & $\begin{array}{l}\text { Musa cv.Goly- } \\
\text { goly pot-pot }\end{array}$ & Australia & JQ743567 & N/A & KF009692 & KF014013 & N/A \\
\hline P. mangifera-indica & CPC 20274 & Mangifera indica & Thailand & KF170305 & KF206240 & KF289190 & KF289296 & KF289121 \\
\hline P. minima & CBS 585.84 & Acer rubrum & USA & KF206176 & KF206286 & KF289204 & KF289249 & KF289135 \\
\hline P. musaechinensis & GZAAS6.1247 & Musa.sp. & China & KF955294 & N/A & KM816639 & KM816627 & KM816633 \\
\hline & GZAAS6.1384 & Musa sp. & China & KF955295 & N/A & KM816640 & KM816628 & KM816634 \\
\hline P. musarum & BRIP57803 & Musa sp. & Malaysia & JX997138 & N/A & KF009737 & KF014055 & N/A \\
\hline & BRIP58028 & Musa sp. & Australia & KC988377 & N/A & KF009738 & KF014054 & N/A \\
\hline P. neopyrolae & CPC 21879 & Pyrola asarifolia & Japan & AB454318 & AB454241 & N/A & AB704233 & N/A \\
\hline P. owaniana & CBS 776.97 & $\begin{array}{l}\text { Brabejum } \\
\text { stellatifolium }\end{array}$ & South Africa & FJ538368 & KF206293 & FJ538426 & KF289254 & JF343767 \\
\hline & СРC 14901 & $\begin{array}{l}\text { Brabejum } \\
\text { stellatifolium }\end{array}$ & South Africa & JF261462 & KF206303 & JF261504 & KF289243 & JF343766 \\
\hline P. pachysandricola & MUCC 124 & $\begin{array}{l}\text { Pachysandra } \\
\text { terminalis }\end{array}$ & Japan & AB454317 & AB454317 & N/A & AB704232 & N/A \\
\hline
\end{tabular}




\begin{tabular}{|c|c|c|c|c|c|c|c|c|}
\hline P. parthenocissi & CBS111645 & $\begin{array}{l}\text { Parthenocissus } \\
\text { quinquefolia }\end{array}$ & USA & EU683672 & N/A & JN692530 & JN692518 & N/A \\
\hline \multirow[t]{2}{*}{ P. partricuspidatae } & NBRC9466 & $\begin{array}{l}\text { Parthenocissus } \\
\text { tricuspidata }\end{array}$ & Japan & KJ847424 & N/A & KJ847446 & KJ847432 & KJ847440 \\
\hline & NBRC9757 & $\begin{array}{l}\text { Parthenocissus } \\
\text { tricuspidata }\end{array}$ & Japan & KJ847425 & N/A & KJ847447 & KJ847433 & KJ847441 \\
\hline P. paxistimae & CBS 112527 & $\begin{array}{l}\text { Paxistima } \\
\text { mysinites }\end{array}$ & USA & KF206172 & KF206320 & KF289209 & KF289239 & KF289140 \\
\hline \multirow[t]{2}{*}{ P. philoprina } & CBS 587.69 & Ilex aquifolium & Spain & KF154278 & KF206297 & KF289206 & KF289250 & KF289137 \\
\hline & CBS 616.72 & Ilex aquifolium & Germany & KF154279 & KF206296 & KF289205 & KF289251 & KF289136 \\
\hline P. podocarpicola & CBS 728.79 & Podocarpus maki & USA & KF206173 & KF206295 & KF289203 & KF289252 & KF289134 \\
\hline \multirow[t]{2}{*}{ P. podocarpi } & CBS 111646 & $\begin{array}{l}\text { Podocarpus } \\
\text { falcatus }\end{array}$ & South Africa & AF312013 & KF206323 & KC357671 & KC 357670 & KF289169 \\
\hline & CBS 111647 & $\begin{array}{l}\text { Podocarpus } \\
\text { lanceolata }\end{array}$ & South Africa & KF154276 & KF206322 & KF289232 & KF289235 & KF289168 \\
\hline P. pseudotsugae & CBS 111649 & $\begin{array}{l}\text { Pseudotsuga } \\
\text { menziesii }\end{array}$ & USA & KF154277 & KF206321 & KF289231 & KF289236 & KF289167 \\
\hline P. rhaphiolepidis & MUCC 432 & $\begin{array}{l}\text { Rhaphiolepis } \\
\text { indica }\end{array}$ & Japan & DQ632660 & N/A & N/A & AB704242 & N/A \\
\hline P. rubra & CBS 111635 & Acer rubrum & USA & KF206171 & EU754194 & KF289198 & KF289233 & KF289129 \\
\hline P. schimae & $\begin{array}{l}\text { CGMCC } \\
3.14354\end{array}$ & Schima superba & China & JN692534 & N/A & JN692522 & JN692510 & JN692506 \\
\hline \multirow[t]{2}{*}{ P. schimicola } & $\begin{array}{l}\text { CGMCC } \\
3.17319\end{array}$ & Schima superba & China & KJ847426 & N/A & KJ847448 & KJ847434 & KJ854895 \\
\hline & $\begin{array}{l}\text { CGMCC } \\
3.17320\end{array}$ & Schima superba & China & KJ847427 & N/A & KJ847449 & KJ847435 & KJ854896 \\
\hline P. speewahensis & BRIP 58044 & Orchids & Australia & KF017269 & N/A & KF017268 & N/A & N/A \\
\hline P. sphaeropsoidea & CBS 756.70 & $\begin{array}{l}\text { Aesculus } \\
\text { hippocastanum }\end{array}$ & Germany & AY042934 & KF206294 & KF289202 & KF289253 & KF289133 \\
\hline P. spinarum & CBS 292.90 & $\begin{array}{l}\text { Chamaecyparis } \\
\text { pisifera }\end{array}$ & France & JF343585 & KF206301 & JF343606 & JF343669 & JF343773 \\
\hline \multirow[t]{2}{*}{ P. styracicola } & $\begin{array}{l}\text { CGMCC } \\
3.14985\end{array}$ & $\begin{array}{l}\text { Styrax } \\
\text { gradiflorus }\end{array}$ & China & JX052040 & N/A & JX025045 & JX025036 & KF289141 \\
\hline & CGMCC & Styrax & China & JX052041 & N/A & JX025046 & KF289255 & KF289165 \\
\hline
\end{tabular}




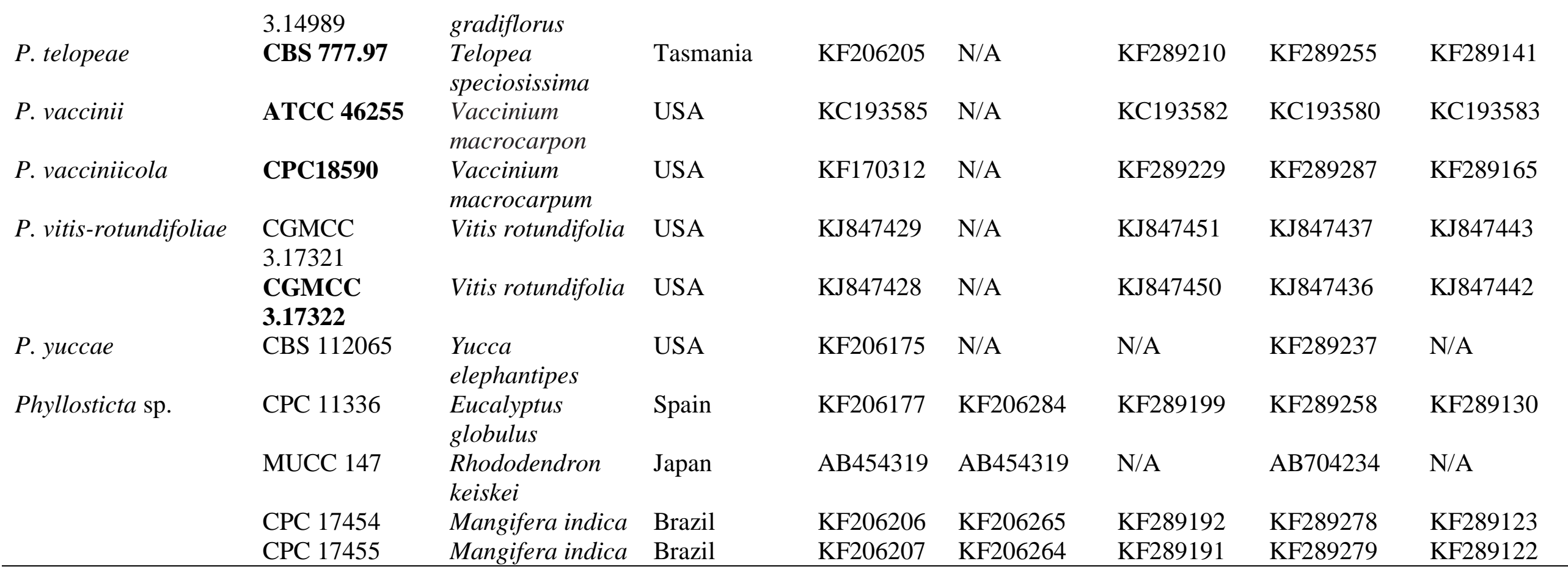

a. New species described and strains sequenced in this study are shown in bold.

b. ATCC: American Type Culture Collection, Virginia, USA; BRIP: Plant Pathology Herbarium, Biosecurity Queensland, Dutton Park, Queensland, Australia; CBS: CBS-KNAW Fungal Biodiversity Centre, Utrecht, the Netherlands; CGMCC: China, General Microbiological Culture Collection, Beijing, China; CPC: Culture collection of P.W. Crous, housed at CBS; GZAAS : Guizhou Academy of Agricultural Sciences Collection , China; IMI: International Mycological Institute, CABI-Bioscience, Egham, Bakeham Lane, U.K.; LGMF: Culture collection of Laboratory of Genetics of Microorganisms, Federal University of Parana, Curitiba, Brazil; MAFF: the Microbiological Genbank, National Institute of Agrobiological Sciences, Japan; MUCC: Culture Collection, Laboratory of Plant Pathology, Mie University; NBRC: Biological Resource Center, the National Institute of Technology, and Evaluation, Japan; ZJUCC: Zhejiang University Culture Collection, China.

c. Type and ex-type cultures are in bold. 


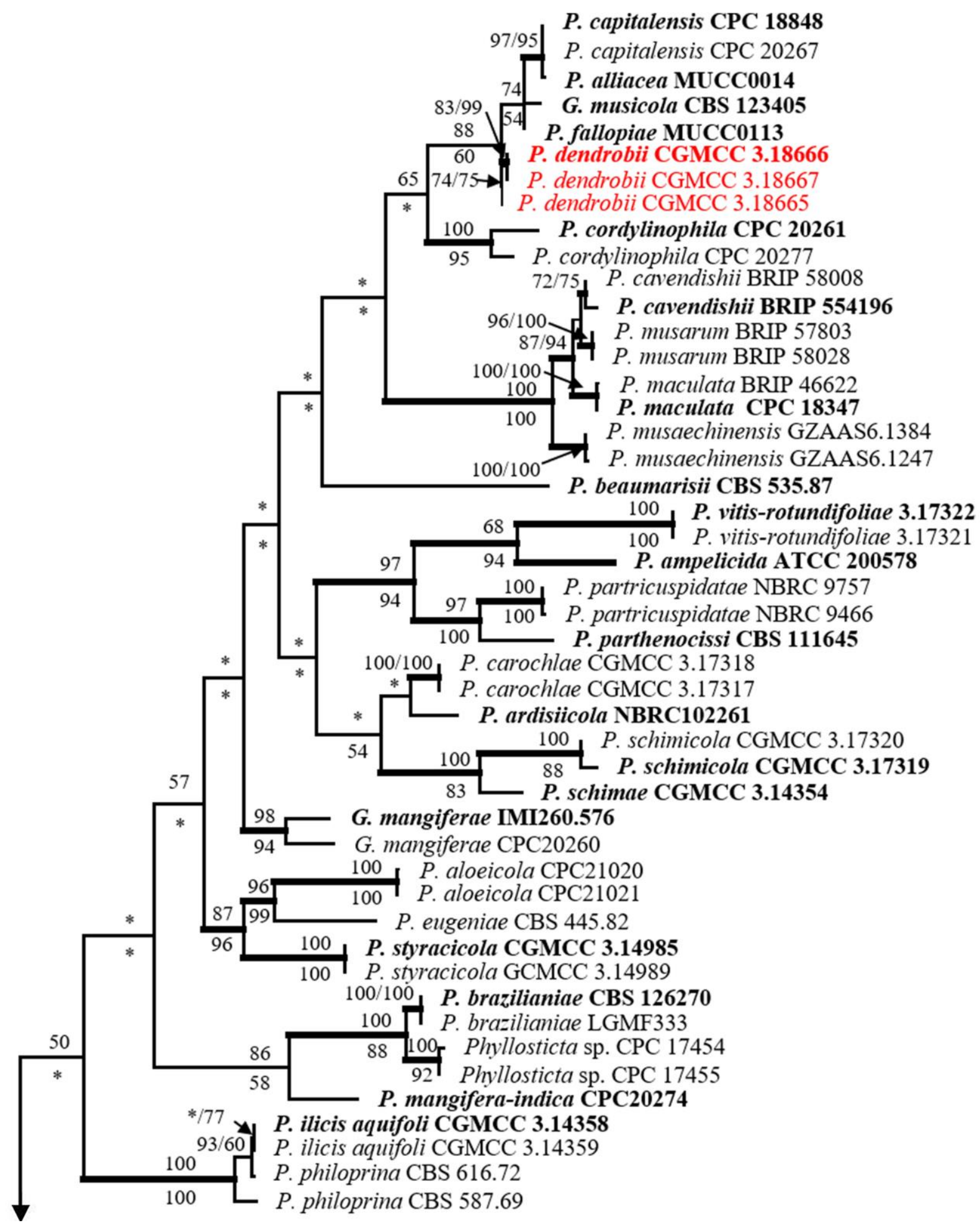

Figure 1 - Maximum parsimony tree generated from sequence analysis of the combined ITS, LSU, tefl-a, ACT and GPDH dataset. Bootstrap support values for maximum parsimony (MP) equal to or greater than $50 \%$ are shown above the nodes. Bootstrap support values for maximum likelihood (ML) equal to or greater than 50\% are shown under the nodes. Bayesian bootstrap (BP) posterior probability scores above 0.90 are indicated as bold branches. Bootstrap support values lower than $50 \%$ for MP and ML are indicated with an asterisk. The species characterized in this study are in red, and the ex-type strains are in boldface. 


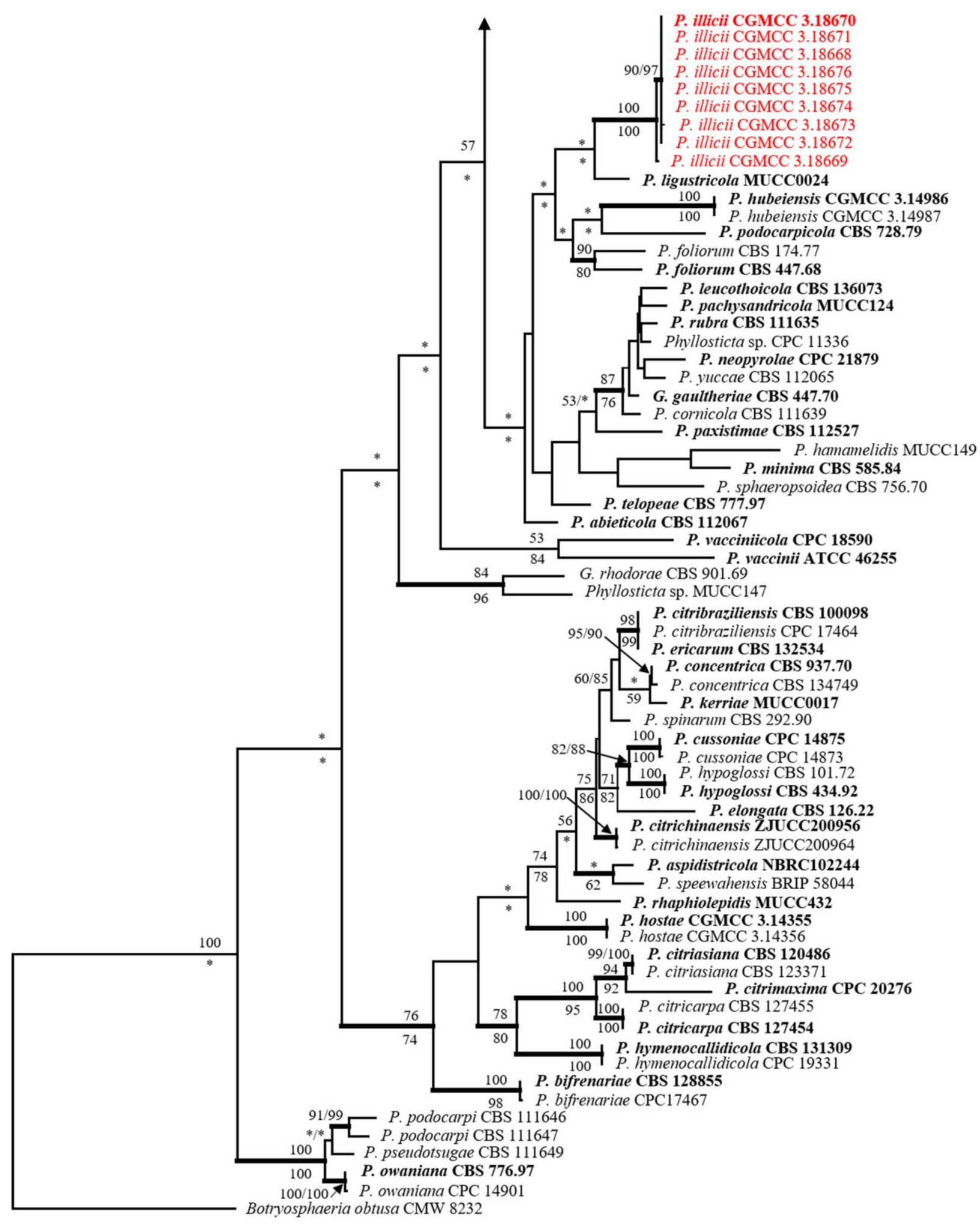

10

Figure 1 - (continued).

Culture characteristics: Colonies erumpent, spreading, with sparse aerial mycelium and feathery margins, diameter up to $31 \mathrm{~mm}$ after 1 week and $68 \mathrm{~mm}$ after 2 weeks at $28^{\circ} \mathrm{C}$. On MEA surface olivaceous-grey, reverse iron-grey. 

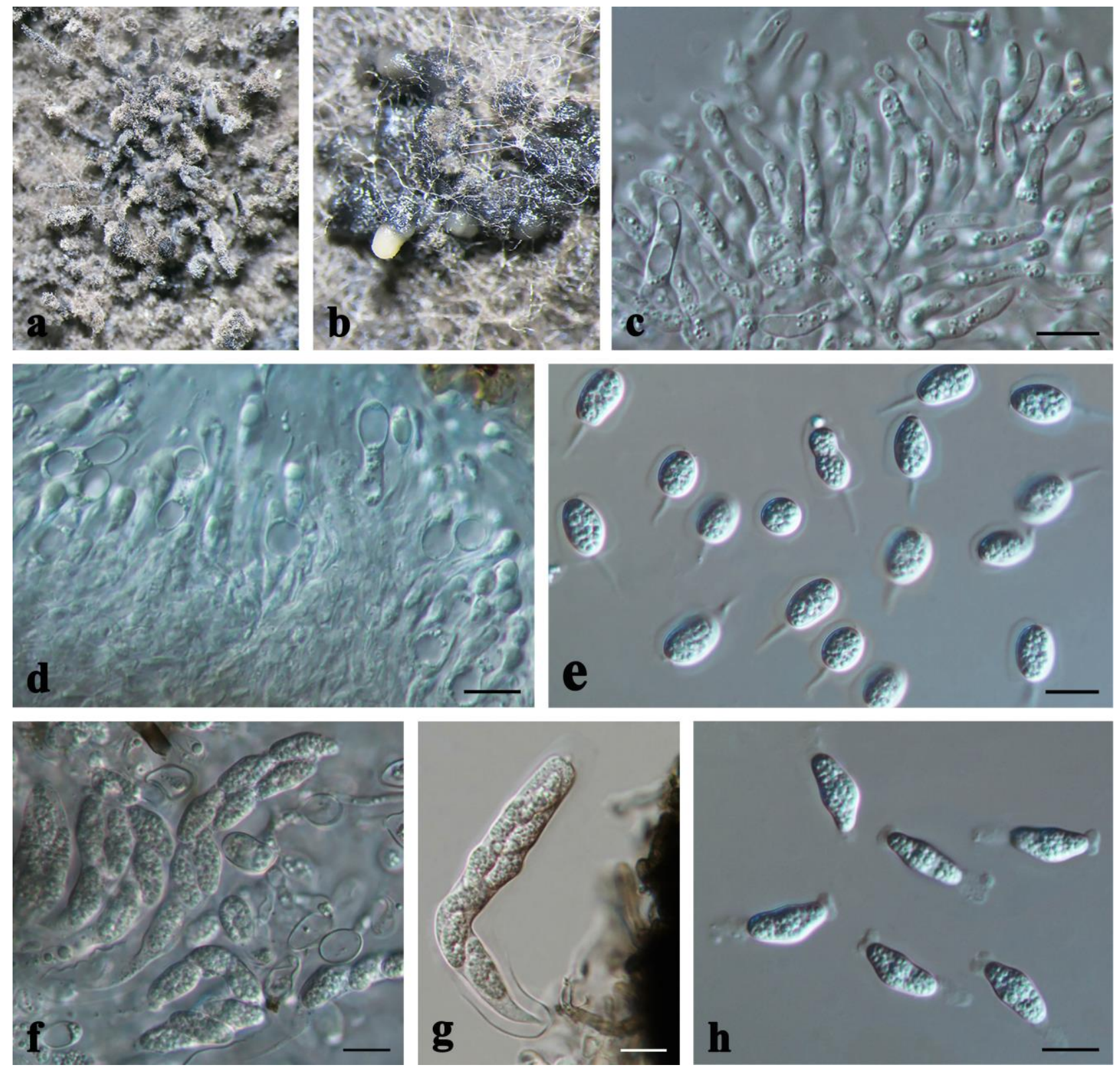

Figure 2 - Phyllosticta dendrobii (HMAS255228, holotype). a, b Conidiomata on MEA. c initial conidiogenous cells. d conidia developing on conidiogenous cells. e conidia. $f, g$ ascomata. $h$ ascospores. - Scale bars: $\mathrm{c}-\mathrm{h}=10 \mu \mathrm{m}$.

Specimen examined - CHINA, Jiangxi province, associated from Dendrobium nobile, 9 October, 2014, leg. X. Sun (HMAS255228, holotype); CGMCC 3.18666 (ex-type), CGMCC 3.18665; CGMCC 3.18667.

Notes - The subclade consists of $P$. alliacea Motohashi, Jun. Nishikawa \& C. Nakash., $P$. capitalensis, P. fallopiae Motohashi, I. Araki \& C. Nakash., Guignardia musicola Wulandari, L. Cai \& K.D. Hyde and P. dendrobii received high bootstrap support with $P$. dendrobii basal to all other species (Fig 1). The conidia of Phyllosticta are mostly 10-25 $\mu \mathrm{m}$ long (Wikee et al. 2011), while the small conidia of $P$. dendrobii can be easily distinguishable from most reported species of Phyllosticta. Morphologically, $P$. dendrobii is comparable with P. aplectri, which was reported from Aplectrum hyemale (Orchidaceae). Although the condial dimensions of $P$. dendrobii and $P$. aplectri are similar $(5-8 \times 4-6 \mu \mathrm{m} v s .(5-) 8-9(-10) \times 5(-7) \mu \mathrm{m})$, the pycnidia of $P$. dendrobii are larger those of $P$. aplectri (up to $280 \mu \mathrm{m} v$ s. 65-90 $\mu \mathrm{m}$ ). Phyllosticta dendrobii is the first Phyllosticta species reported from Dendrobium. 
Phyllosticta illicii S. Lin, Y. Zhang ter, sp. nov.

MycoBank: 821785; Facesoffungi number: FoF03423

Etymology - Named after the host genus from which it was collected, Illicium verum.

Conidiomata pycnidial, mostly aggregated in clusters, black, erumpent, globose to clavate or elongated with necks up to $500 \mu \mathrm{m}$ long, exuding colourless to opaque conidial masses. Pycnidia up to $200 \mu \mathrm{m}$ diam. Pycnidial wall of several layers of textura angularis, up to $40 \mu \mathrm{m}$ thick, inner wall of hyaline textura angularis. Ostiole central, up to $25 \mu \mathrm{m}$ diam. Conidiophores reduced to conidiogenous cells, Conidiogenous cells terminal, subcylindrical, hyaline, smooth, 7-13 × 3-5 $\mu \mathrm{m}$; proliferating several times percurrently near apex. Conidia $(12-) 13-15(-17) \times 7(-9) \mu \mathrm{m}$, solitary, hyaline, aseptate, thin and smooth walled, coarsely guttulate, or with a single large central guttule, ovoid to irregularly ellipsoid, enclosed in a thin mucoid sheath, $1 \mu \mathrm{m}$ thick, and bearing a hyaline apical mucoid appendage, $(6-) 18-35(-36) \times 1(-2) \mu \mathrm{m}$, flexible, unbranched, tapering towards an acutely rounded tip. After 2 months, mucoid sheath and apical mucoid appendage disappear. Spermatia occurring in the same conidioma with conidia, hyaline, smooth, guttulate to granular, bacilliform, $7-13 \times 2-3 \mu \mathrm{m}$.

Culture characteristics - Colonies erumpent, spreading, with sparse aerial mycelium and feathery margins, diameter up to $33 \mathrm{~mm}$ after 1 week and $76 \mathrm{~mm}$ after 2 weeks at $28^{\circ} \mathrm{C}$. On MEA surface olivaceous-grey, reverse iron-grey.

Specimen examined - CHINA, Guangxi Province, associated from Illicium verum, 9 October 2014, leg. X. Sun (HMAS 255230, holotype), CGMCC 3.18670 (ex-type), CGMCC3.18668, CGMCC3.18669, CGMCC3.18671, CGMCC3.18672, CGMCC3.18673, CGMCC3.18674, CGMCC3.18675, CGMCC3.18676.

Notes - Phylogenetically, the sub clade comprised of $P$. illicii is well supported, and sibling to other species of Phyllosticta (Fig 1). Morphologically, the long apical mucoid appendage is distinguishable from most other species of Phyllosticta (mostly shorter than $15 \mu \mathrm{m}$, van der Aa 1973, van der Aa \& Vanev 2002). Phyllosticta illicii is the first Phyllosticta species reported from Illicium.
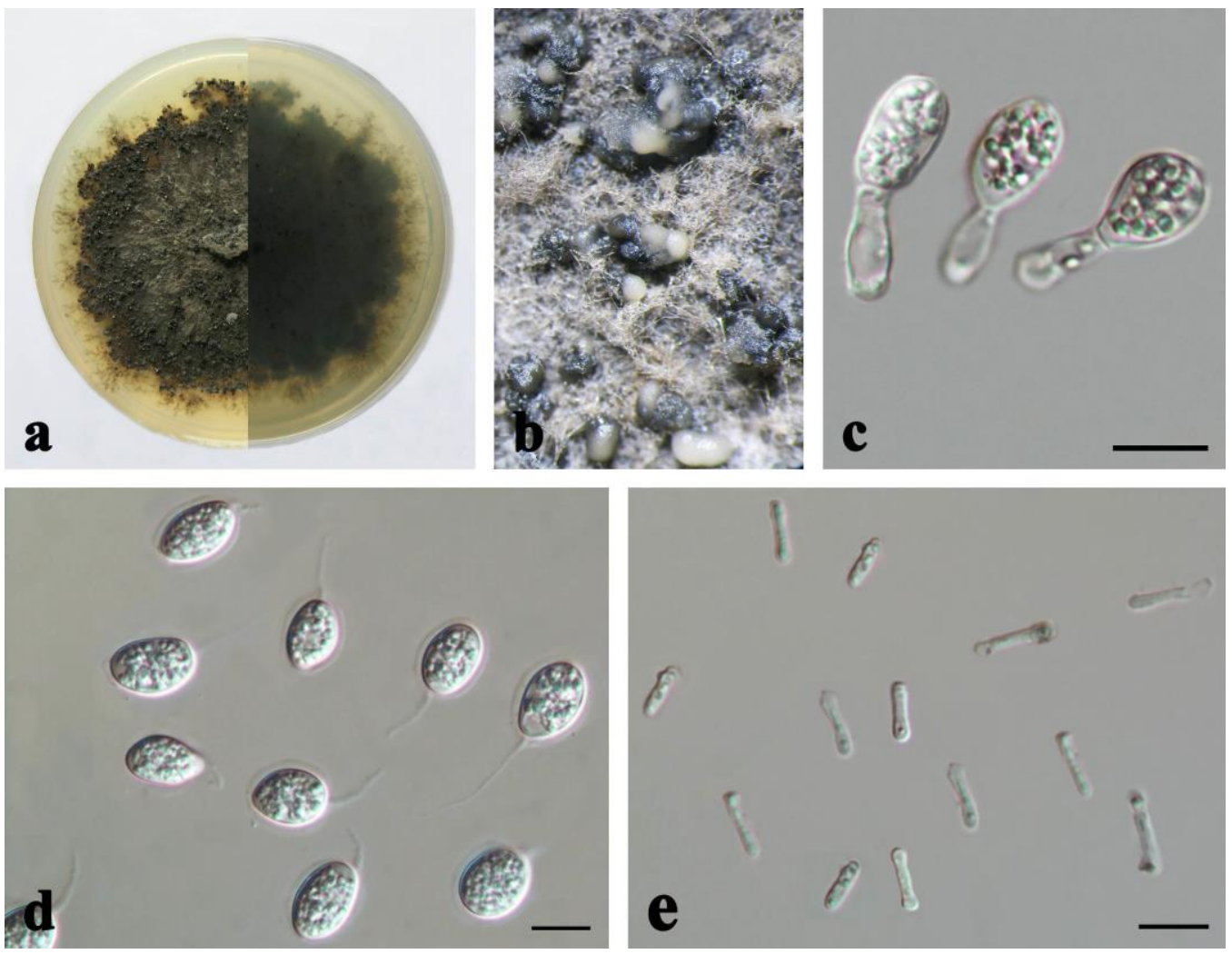

Figure 3 - Phyllosticta illicii (HMAS 255230, holotype). a, b Conidiomata on MEA. c Conidia developing on conidiogenous cells. $\mathrm{d}$ Conidia. e Spermatia. - Scale bars: $\mathrm{c}-\mathrm{e}=10 \mu \mathrm{m}$. 


\section{Discussion}

The morphological characteristics of $P$. dendrobii and $P$. illicii, such as aseptate, hyaline conidia that are usually covered by a mucoid layer and bearing a single apical appendage of fit well within Phyllosticta well (van der Aa 1973, van der Aa \& Vanev 2002). The long apical appendage of $P$. illicii and small conidia of $P$. dendrobii differ from most reported species of Phyllosticta.

Both $P$. dendrobii and $P$. illicii were isolated as endophytic fungi from healthy tissues of Dendrobium nobile and Illicium verum respectively in this study. Several other species of Phyllosticta have been reported as endophytes, such as $P$. capitalensis, which is a cosmopolitan endophytic species reported on more than 20 hosts in eight countries (Okane et al. 2001, Baayen et al. 2002, Okane et al. 2003, Glienke et al. 2011). Phyllosticta bifrenariae and P. brazilianiae were reported as endophytes on Bifrenaria (Orchidaceae) and Mangifera indica (Anacardiaceae) respectively in Brazil (Glienke et al. 2011). Phyllosticta ilicina and P. spinarum were isolated from healthy leaves of Quercus ilex (Fagaceae) and Platycladus orientalis (Cupressaceae) respectively (Collado et al. 1996, Wijeratne et al. 2008). The relationships between hosts and entophytes, however, can be mutualistic, symbiotic, antagonistic or neutral, and may turn pathogenetic during host senescence (Hyde \& Soytong 2008, Rodriguez \& Redman 2008, Rodriguez et al. 2009, Aly et al. 2011).

Orchid seems a common host for Phyllosticta, and several species have been reported from plants of Orchidaceae. Phyllosticta capitalensis and P. bifrenariae were reported on Stanhopea and Bifrenaria (Orchidaceae) respectively in Brazil, and P. speewahensis from Vanda (Waxy Blue, Orchidaceae) in Australia (Hennings 1908, Glienke et al. 2011, Shivas et al. 2013). Host association has been used in species identification of Phyllosticta, and this has been proved to be unacceptable (van der Aa 2002). For instance, there are six species of Phyllosticta associated with Citrus spp., namely P. capitalensis, P. citricarpa, P. citriasiana Wulandari, Crous \& Gruyter, $P$. citrimaxima S. Wikee, Crous, K.D. Hyde \& McKenzie, $P$. citribraziliensis O.L. Pereira, Glienke \& Crous and $P$. citrichinaensis X.H. Wang, Chen, Huang, Zhang, K.D. Hyde \& H.Y. Li. Of these $P$. capitalensis can be associated with various host plants (Wikee et al. 2013). The host specificity of Phyllosticta is not clear yet, while it may depend on the particular species or their life style. Some species that cause plant diseases show host specificity, while the entophytic ones tend to be generalists (Wikee et al. 2013).

\section{Acknowledgements}

This study was financially supported by NSFC Projects of National Natural Science Foundation of China (General Program, 31770015, 31370063), International Cooperation and Exchanges (31461143028) and National Science and Technology Foundation Project (2014FY210400).

\section{References}

Allescher A. 1898 - Rabenhorst's Kryptogamen-flora, Pilze-Fungi imperfecti. E. Kummer, Leipzig, Germany.

Aly AH, Debbab A, Proksch P. 2011 - Fungal endophytes: unique plant inhabitants with great promises. Applied Microbiology and Biotechnology 90, 1-17.

Baayen R, Bonants P, Verkley GJM, Carroll GC et al. 2002 - Nonpathogenic isolates of the citrus black spot fungus, Guignardia citricarpa, identified as a cosmopolitan endophyte of woody plants, G. mangiferae (Phyllosticta capitalensis). Phytopathology 92, 464-477.

Carbone I, Kohn LM. 1999 - A method for designing primer sets for speciation studies in filamentous ascomycetes. Mycologia 1999, 553-556.

Collado J, Platas G, Peláez F. 1996 - Fungal endophytes in leaves, twigs and bark of Quercus ilex from Central Spain. Nova Hedwigia 63, 347-360.

Crous PW, Gams W, Stalpers JA, Robert V et al. 2004 - MycoBank: an online initiative to launch mycology into the 21st century. Studies in Mycology 50, 19-22. 
Fries EM. 1849 - Summa vegetabilium Scandinaviae. Typographis Academica, Uppsala.

Glienke C, Pereira O, Stringari D, Fabris J et al. 2011 - Endophytic and pathogenic Phyllosticta species, with reference to those associated with Citrus Black Spot. Persoonia 26, 47-56.

Grove WB 1935 - British stem-and leaf-fungi (Coelomycetes) 1. University Press, Cambridge, England.

Guerber JC, Liu B, Correll JC, Johnston PR. 2003 - Characterization of diversity in Colletotrichum acutatum sensu lato by sequence analysis of two gene introns, mtDNA and intron RFLPs, and mating compatibility. Mycologia 95, 872-895.

Hennings PC. 1908 - Fungi S. Paulenses IV. a cl. Puttemans collecti. Hedwigia 48, 1-20.

Huelsenbeck JP, Ronquist F. 2005 - Bayesian analysis of molecular evolution using MrBayes, pp. 183-232 in Statistical Methods in Molecular Evolution, edited by R. Nielsen. Springer, New York.

Hyde KD, Soytong K. 2008 - The fungal endophyte dilemma. Fungal Diversity 33, 163-173.

Jayasiri SC, Hyde KD, Ariyawansa HA, Bhat J et al. 2015 - The Faces of Fungi database: fungal names linked with morphology, phylogeny and human impacts. Fungal Diversity 74, 3-18 (DOI 10.1007/s13225-015-0351-8).

Michalak S. 2012 - raxmlGUI: a graphical front-end for RAxML. Organisms Diversity and Evolution 12, 335-337.

Moncalvo JM, Wang HH, Hseu RS. 1995 - Phylogenetic relationships in Ganoderma Inferred from the Internal Transcribed Spacers and 25S Ribosomal DNA Sequences. Mycologia 87, 223238.

Myllys L, Stenroos S, Thell A 2002 - New genes for phylogenetic studies of lichenized fungi: glyceraldehyde-3-phosphate dehydrogenase and beta-tubulin genes. Lichenologist 34, $237-$ 246.

Okane I, Lumyong S, Nakagiri A, Ito T. 2003 - Extensive host range of an endophytic fungus, Guignardia endophyllicola (anamorph: Phyllosticta capitalensis). Mycoscience 44, 353-363.

Okane I, Nakagiri A, Ito T. 2001 - Identity of Guignardia sp. inhabiting ericaceous plants. Canadian Journal of Botany 79, 101-109.

Page RD. 1996 - TreeView: an application to display phylogenetic trees on personal computers. Computer Applications in the Biossciences 12, 357-358.

Persoon CH. 1818 - Traité sur les champignons comestibles, contenant l'indicationdes espèces nuisibles; a l'histoire des champignons. Belin-Leprieur, Paris, France.

Posada D, Buckley TR. 2004 - Model selection and model averaging in phylogenetics: advantages of Akaike information criterion and Bayesian approaches over likelihood ratio tests. Systematic Biology 53, 793-808.

Pu J, Xie Y, Zhang X, Qi Y et al. 2008 - Preinfection behaviour of Phyllosticta musarum on banana leaves. Australasian Plant Pathology 37, 60-64.

Rodriguez RJ, Redman RS. 2008 - More than 400 million years of evolution and some plants still can't make it on their own: plant stress tolerance via fungal symbiosis. Journal of Experimental Botany 59, 1109-1114.

Rodriguez RJ, White JJ, Arnold AE, Redman RS. 2009 - Fungal endophytes: diversity and functional roles. New Phytologist 182, 314-330.

Ronquist F, Huelsenbeck JP. 2003 - MrBayes 3: Bayesian phylogenetic inference under mixed models. Bioinformatics 19, 1572-1574.

Seaver FJ. 1922 - Phyllostictaceae. North American Flora 6, 3-84.

Shivas RG, Tan YP, Grice KR. 2013 - Phyllosticta species on orchids (Orchidaceae), introducing Phyllosticta speewahensis sp. nov. (Phyllostictaceae, Ascomycota) from northern Australia. Sydowia 65, 139-146.

Sultan A, Johnston PR, Park D, Robertson AW 2011 - Two new pathogenic ascomycetes in Guignardia and Rosenscheldiella on New Zealand's pygmy mistletoes (Korthalsella: Viscaceae). Studies in Mycology 68, 237-247. 
Swofford DL 2002 - PAUP*. Phylogenetic Analysis Using Parsimony (*and Other Methods). Version 4. Sinauer Associates, Sunderland, Massachusetts.

Tamura K, Stecher G, Peterson D, Filipski A et al. 2013 - MEGA6: molecular evolutionary genetics analysis version 6.0. Molecular Biology \& Evolution 30, 2725-2729.

van der Aa HA, Vanev S 2002 - A revision of the species described in Phyllosticta. Utrecht, The Netherlands: Centraalbureau voor Schimmelcultures(CBS).

van der Aa HA. 1973 - Studies in Phyllosticta. Study in Mycology. 5, 1-110.

White TJ, Bruns T, Lee S, Taylor J 1990 - Amplification and direct sequencing of fungal ribosomal RNA genes for phylogenetics. In: PCR Protocols: a guide to methods and applications (Innis MA, Gelfand DH, Sninsky JJ, White TJ, eds). Academic Press, San Diego, California. 18, 315-322.

Wicht B, Petrini O, Jermini M, Gessler C et al. 2012 - Molecular, proteomic and morphological characterization of the ascomycete Guignardia bidwellii, agent of grape black rot: a polyphasic approach to fungal identification. Mycologia 104, 1036-1045.

Wijeratne EMK, Paranagama PA, Marron MT, Gunatilaka MK et al. 2008 - Sesquiterpene quinones and related metabolites from Phyllosticta spinarum, a fungal strain endophytic in Platycladus orientalis of the Sonoran Desert (1). Journal of Natural Products 71, 218-222.

Wikee S, Lombard L, Crous PW, Nakashima C et al. 2013 - Phyllosticta capitalensis, a widespread endophyte of plants. Fungal Diversity 60, 91-105.

Wikee S, Udayanga D, Crous PW, Chukeatirote E et al. 2011 - Phyllosticta an overview of current status of species recognition. Fungal Diversity 51, 43-61.

Wong MH, Crous PW, Henderson J, Groenewald JZ et al. 2012 - Phyllosticta species associated with freckle disease of banana. Fungal Diversity 56, 173-187.

Zhang Y, Jeewon R, Fournier J, Hyde KD 2008 - Multi-gene phylogeny and morphotaxonomy of Amniculicola lignicola: a novel freshwater fungus from France and its relationships to the Pleosporales. Mycological Research 112, 1186-1194. 